\title{
Large-Scale PIV Surface Flow Measurements in Shallow Basins with Different Geometries
}

\author{
Kantoush, S. A.* ${ }^{1,2}$ and Schleiss, A. J.*3
}

\begin{abstract}
*1 Dr., Water Resources Research Center, Disaster Prevention Research Institute, Kyoto University, Goka-sho, Uji-shi, 611-0011, Japan, E-mail: sameh.kantoush@a3.epfl.ch

*2 Swiss National Fund for Scientific Research (SNSF), Bern, Switzerland

*3 Professor, Director, Ecole Polytechnique Fédérale de Lausanne (EPFL), Laboratory of Hydraulic Constructions (LCH), Station 18, CH -1015 Lausanne, Switzerland, E-mail: anton.schleiss@epfl.ch
\end{abstract}

\begin{abstract}
Shallow depth flow fields and low velocity magnitudes are often challenges for traditional velocity measuring instruments. As such, new techniques have been developed that provide more reliable velocity measurements under these circumstances. In the present study, the two-dimensional (2D) surface velocity field of shallow basins is assessed by means of Large-Scale Particle Image Velocimetry (LSPIV). The measurements are carried out at the water surface, which means that a laser light sheet is not needed. Depending on the time scales of the flow and the camera characteristics, it is even possible to work with a constant light source. An experimental application of this method is presented to analyze the effects of shallow basin geometry on flow characteristics in reservoirs where large coherent two-dimensional flow structures in the mixing layer dominate the flow characteristics. The flow and boundary conditions that give rise to asymmetric flow are presented. Asymmetric flow structures were observed starting from basin shape ratios that are less than or equal to 0.96. By decreasing the basin length and increasing the shape ratio to greater than 0.96 , the flow structure generally tends towards a symmetric pattern.
\end{abstract}

Keywords : Visualization, LSPIV, Flow in shallow basins, Basin geometry, Flow patterns.

\section{Introduction}

Shallow flows can be defined as predominantly horizontal flows in a fluid domain where the vertical dimension is significantly smaller than the two horizontal dimensions (Yuce and Chen, 2003). In fact, lakes, reservoirs, most rivers, estuarial and coastal flows fall into this category. The instability of a two dimensional flow in a symmetric shallow reservoir with a suddenly expanded section leads to large-scale transverse motions as a result of the growth of transverse disturbances. It is important to understand such flows because of their prominence in nature and their practical importance in many applications, such as in sudden expansions (Shapira et al., 1998), jets and wakes (Chen and Jirka 1997), storage chambers (Adamsson et al., 2003) and shallow reservoirs (Kantoush et al., 2005, 2006, 2007, 2008, and 2009). Moreover, these large-scale motions influence processes, such as suspended sediment transport, and thus are likely to affect bed deposition patterns. In shallow flow problems, velocity information with high spatial and temporal resolution is needed to understand the dynamics of the flow and the related mixing processes. In the present investigation, a rectangular, triangular 
and hexagonal cavity type flow is considered with the objective of looking at the physical processes involved, particularly the interaction of the large coherent eddy structures in the separated layer with the re-circulating flow. Moreover, to measure the flow characteristics at the surface of shallow water flows over large basins with different geometries, a simple and reliable method has been developed using the advantages of Particle Image Velocimetry (PIV).

Particle image velocimetry (PIV) is a powerful flow field display and analysis technique that measures the velocity field distribution of the flow with a high precision. The basics of the conventional image velocimetry techniques are discussed in detail by Adrian (1991) and Raffel (1997) For instance, planar in-water column flow measurements obtained by PIV are described by Adrian (1991). PIV offers a simple method for surface measurement in areas with complicated geometries and flow conditions. Extension of the conventional image velocimetry to free-surface velocity measurements on large-scale images, commonly termed Large Scale Particle Image Velocimetry (LSPIV), has been recently tested by several investigators, i.e., Weitbrecht et al.(2002) and Fujita et al. (1994, 1998). These studies proved that LSPIV is more reliable in measuring surface flow velocity in experimental flumes and natural rivers compared to conventional techniques (Muste et al., 2004). In hydraulic engineering, this technique has so far been applied mainly for surface velocity measurements of water and ice flow in very uniform flow fields, as well as in groin field experiments (Ettema et al., 1997; Fujita et al., 1998; Weitbrecht et al., 2002; Uijttewaal et al., 2001). LSPIV is an extension of conventional PIV for velocity measurements in large scale flows (Fujita et al., 1998). While the image and data processing algorithms are similar to those used in conventional PIV, adjustments are required for illumination, seeding, and pre-processing of the recorded images.

In LSPIV, the surface flow is seeded with tracer particles that disperse and track the motion of the fluid. If the particles are of suitable size, they will accurately follow the flow and can be considered representative elements of the fluid. In the present study, lighter particles are distributed manually on the flow free surface. These seed particles are illuminated by a light source; usually a laser sheet in conventional image velocimetry is used within the water body, and the light scattered by the particles is collected by a camera. Two images are captured in very rapid succession, and the resulting images can be correlated using the known time interval between images for capturing traveling particles to calculate two components of the representative velocity vectors for each particle. These velocity vectors, when taken across the entire image area, provide a velocity vector map that represents the flow within the illumination plane.

In the framework of a research project, the influence of the large shallow basin geometry on the flow characteristics is presented. The present article describes a simple method developed using the advantage of PIV. Moreover, measurements are presented where the system has been applied to a laboratory problem in order to analyze surface flow in shallow basins of various shapes. In addition to the determination of mean flow conditions and turbulent flow characteristics, this method makes it possible to track two-dimensional large coherent structures, which are the dominant flow phenomena in many shallow flow applications. In these experiments, symmetric and asymmetric flow processes of various shallow geometries have been investigated to determine the influence of these shapes on the transport characteristics of the basin. One of the primary objectives of these experiments is to examine the impact that variation in the geometry shape parameters has on the flow. In this regard, flow pattern, vorticity, and streamlines are important since correct prediction of these features is a necessary condition for matching the phase averaged flow profiles in the experiment.

\section{LSPIV System}

\subsection{Elements of the LSPIV system}

The LSPIV system used in this study was comprised of six elements: a camera, tracer particles, a tracer distributer, an illumination system, a computer for data acquisition as well as analysis, and software (images recording, pre-processing, and post-processing with PIV packages). Depending on the flow velocity, a suitable camera characteristic has been chosen to record images of the flow at a 
suitable rate with adequate image resolution. The sampling frequency of the recorded images has a significant influence on the accuracy of the LSPIV velocity estimates. A CMOS SUMIX-155M camera with a USB2.0 interface and a gray scale resolution of 12 bits was used to capture images of tracer particles on the surface of the model. The camera is a 1.3 mega-pixel (1280 x 1024) CMOS active imaging pixels camera that can record up to 33 frames per second. The SUMIX has a number of qualities that make it well suited for PIV: (1) high resolution images, (2) a high quality monochrome mode, (3) ability to detect low light levels, (4) remote control by a computer, (5) directly downloadable images and (6) relatively low cost (less than $\$ 1,000$ ). The time between images belonging to an image pair can be as small as $0.2 \mathrm{~s}$. Although the SUMIX has a maximum frame rate of 33 frames per second, lower frame rates can be used, or consecutive images can be skipped, to achieve longer separation times. Camera shutter speeds were usually set higher than $0.02 \mathrm{~s}$ to limit particle motion within the images while maintaining adequate lighting. The time between frames varies with shutter speed and frame rate settings. The flow field was slow enough, and seven velocity fields per second can be obtained. A wide angle lens was used with the SUMIX-155M digital camera in order to capture large measurement planes. While the space above the experiment is limited to a maximum of $3.10 \mathrm{~m}$, a C-Mount, $4.8 \mathrm{~mm}$ wide angle lens (M0518) from Computar ${ }^{\circledR}$ and Ganz ${ }^{\mathrm{TM}}$ brand manufacture with small distortion errors was used. This lens provides very good resolution combined with high luminosity, although limited information about the lens is provided by the manufacturer (which likely never foresaw the use of this camera as a particle velocimetry instrument). Consequently, lens calibrations were performed using the PTLens and FlowManager software. Details of this software can be found in section 2.2. The camera was fixed perpendicularly above the basin, covering the basin area over the whole width of $4.0 \mathrm{~m}$ and $5.0 \mathrm{~m}$ of the length and only missing $0.5 \mathrm{~m}$ from the upstream and downstream ends. Velocity correction methods suggested by Muste et al. (1999) to adjust for image distortion were unnecessary since the optical axis of the camera was perpendicular to the plane of the water surface and image distortion was small. When distortion corrections were applied, it was found that they had only a slight effect on velocity measurements.

Determining which seed particle to use for PIV is very important. The seed particles must be large enough to scatter sufficient light for imaging but small enough to accurately track the fluid flow. In addition, the number of particles in the seeding volume must be addressed so that the spatial resolution of particles provides images suitable for processing. The particles have to fit different requirements to be applicable. In the present case of surface velocity measurements, the tracer particles have to swim at the water surface, so the material has to be somewhat lighter than water. The flow was seeded with white floating cylindrical polypropylenes particles with an average diameter of $3.4 \mathrm{~mm}$ and a specific weight of $960 \mathrm{~kg} / \mathrm{m}^{3}$. In order to minimize agglomeration effects, they were coated with paint. This particle diameter corresponded to about 3.1 pixels, which was sufficient to overcome the problem of peak-locking effects (Weitbrecht et al., 2002) and follow the vortex flow structure. These particles can be purchased from DuPont Polymer Powders Coatings, Switzerland under the name "MP 90, Eraclene". The contrast between the white tracers and the dark background of the model was good. A homogeneous distribution of particles within the measuring field is important for good results in terms of a closed velocity vector field. A tracer distributer that is able to seed the water surface homogeneously with particles, at a rate that depends on the ambient flow velocity has to be developed. At the beginning, the particles were spread manually over the whole basin surface. Then, a manual continuous distribution at the inlet channel was located near the water surface in order to avoid surface waves from falling particles. The particles were recollected at the end of the flow section and reused, as the number of particles that is needed for such an experiment is quite high.

The illumination component proved to be the most crucial in order to get high-quality data. The spots must be placed around or on top of the camera to get a homogenous light intensity distribution over the entire area; otherwise the reflections and shadows will show different characteristics on either side. Furthermore, the number of achievable data differs because unequal amounts of reflections are recorded on either side of the basin. Sometimes the natural light is enough. Several illumination arrangements were tested to obtain good quality recordings. Four lamps were positioned face-to-face on both longitudinal sides of the reservoir. The model was illuminated with 
the laboratory's halogen lamps; no special lighting was necessary. However, lights directly above the camera were turned off to prevent reflections from interfering with measurements.

The appropriate choice of the computer helps to capture process of images so that all recorded images from good sequences are maintained and not lost. The PC must be equipped with at least 1 GB RAM to store the images before shifting them to the hard disk. Therefore, the RAM is the limiting factor for the length of a time series.

The software choice is important in terms of data quality and time expenditure. Special software for PIV analysis of the recorded images uses statistical means to estimate the displacement of flow tracers between images and estimate velocities over the entire imaged area. Two types of software exist: free software (e.g., MatPIV, PIVMat, mpiv, URAPIV, DigiFlow, and PIV Sleuth), and commercial PIV packages (e.g., FlowManager (Dantec dynamics), and DaVis (LaVisions) software).

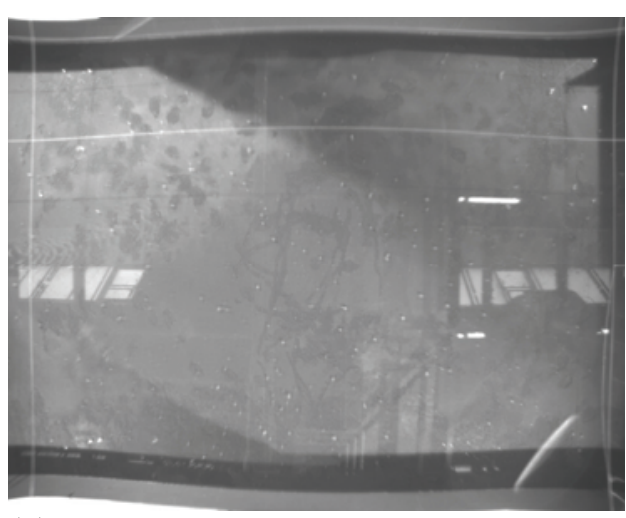

(a)

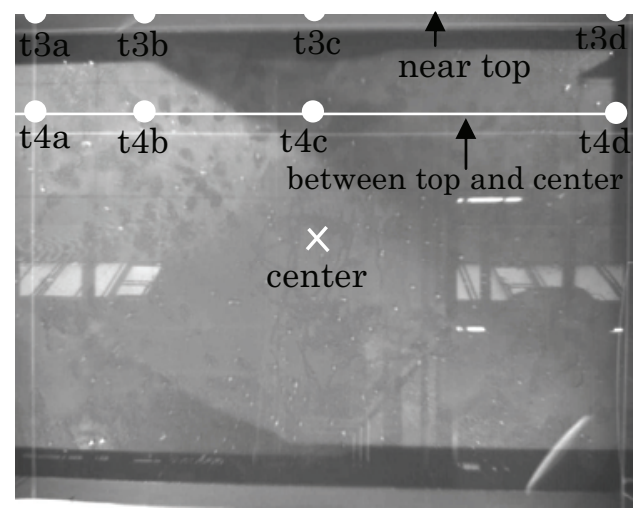

(b)

Fig. 1. (a) Distorted image (b) corrected image with the control points by PTLens

\subsection{Calibration of the camera lens}

For LSPIV applications, wide-angle lenses are most often needed in order to record a large surface. However, every lens produces some distortion on an image captured with a camera. Distortions cause straight lines in the scene to appear as curves in the image. Reconstruction of displacements from the image plane (CMOS-plane) to the object plane (i.e., in physical space) can be numerically performed, provided it is known how 'points' in the object plane are imaged onto the CMOS-plane. Two calibration techniques are considered: (1) the use of PTLens software and (2) Dantec Dynamics FlowManager.

In essence, PTLens provides a simple graphical interface between the user and the Panorama Tools library (pano12.dll) Calibration Toolbox developed by Dersch (2003). To correct distortion, PTLens examines JPEG EXIF information to determine camera make, model, and lens focal length. Then, it uses these parameters to look up lens correction coefficients in a database and calls Panorama Tools to correct the image with details from the PTLens manual (epaperpress.com/ptlens). Figures 1 (a) and (b) are examples of an image distorted by a wide angle lens and the corrected image obtained with the PTLens.

The calibration procedure using PTLens was performed by using a calibration target and a translation stage and by taking pictures at different depths. To calibrate the lens, a target with many straight continuous horizontal features is chosen. For an accurate calibration, two continuous horizontal features that go from edge to edge as shown in Figure 1(b) are required. One feature is placed near the top of the target, and another is placed between the top and the center of the image (Fig. 1(b)). A sufficient distance between the lens and the target is maintained. The target is photographed in landscape mode. Then, calibration points on the features are placed as indicated by the white dots Figure 1(b). The appropriate tab in the PTGui tools is chosen and new line (t3) is specified for control point type and points $(\mathrm{t} 3 \mathrm{a}, \mathrm{t} 3 \mathrm{~d})$ are placed. Then, points $(\mathrm{t} 3 \mathrm{~b}, \mathrm{t} 3 \mathrm{c})$ are added within the existing line. Using a similar procedure, a new t4 line between the top and center is specified, and the points are placed as shown in Figure 1(b). Lines t3 and t4 are treated independently by the optimizer. Care must be taken to ensure that points $\mathrm{b}$ and $\mathrm{c}$ lie within the line 
$(a, d)$. The optimizer will warp the image to bring points b and $c$ in line with line $(a, d)$. Since line (a,d) acts as a base for the points $b$ and $c$, points a and $d$ must be placed symmetrically. That is, the distance between the left edge and point $\mathrm{a}$, and the right edge and point $\mathrm{d}$, should be the same.

With FlowManager, the calibration uses a well-defined grid of dots, which are usually mounted on a traverse system. The calibration target is aligned with the light sheet and normally traversed through the light sheet in several positions. The dots on the calibration target are used to calculate linear or higher order calibration equations for the camera. The linear calibration compensates for differences in scale and lack of orthogonality (e.g., perspective), while higher order calibration equations compensate for non-linear distortions like radial and decentering distortion. In effect, results are calibrated by using the two ways and they have good agreement. For the present paper, a direct linear transform, as explained in Willert (1997), was used for the image plane. The implementation is documented in the software manual for the program Flowmanager (Dantec Dynamics).

\subsection{Accuracy of the measuring system}

A calibration task requires checking the accuracy of the LSPIV system. The accuracy of the LSPIV method was tested using two simple test cases. First, fully developed laminar flow in the straight approach channel with dimensions of $8.0 \mathrm{~m}$ length (the whole length of the installation) and $0.25 \mathrm{~m}$ width was measured. A comparison of the average velocity profile in the main flow direction obtained with LSPIV and numerical simulations at the middle of straight channel is shown in Figure 2(a). Moreover, LSPIV velocities measured by timing hand-released tracers as they moved known distances in the model were tested. The second test of the accuracy of the LSPIV measurements was performed in a rectangular reservoir of 5.0 length and width $3.0 \mathrm{~m}$ by comparing them with UVP (Ultrasonic Doppler Velocity Profiler) measurements and the results of numerical simulations. Figure 2(b) compares the computed and measured values of axial velocity at the centerline of the basin. Velocity distributions for UVP and LSPIV are approximately the same in the approach channel. More details about comparing numerical simulation and measured data can be found in Dewals et al. (2008).

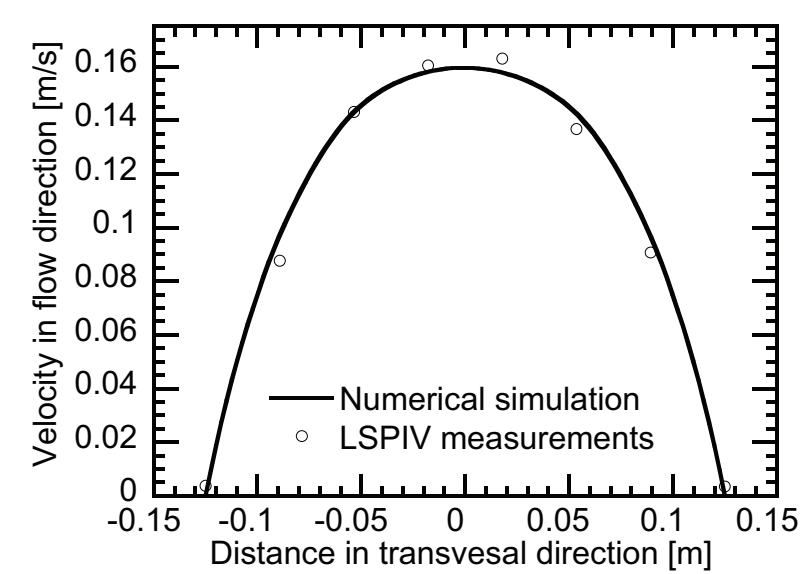

(a) Cross section at the middle of straight channel

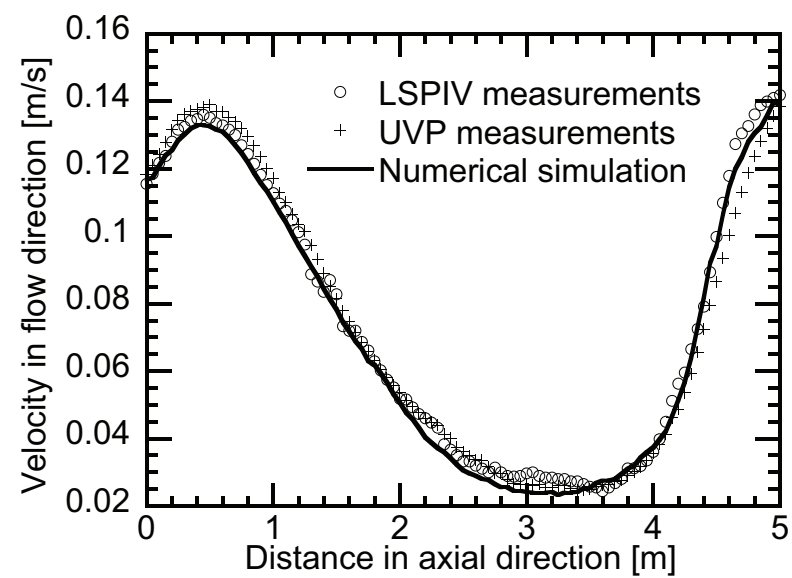

(b) Along the centerline of the reservoir

Fig. 2. Comparison of LSPIV, UVP, and numerical simulation velocities for calibration task

\subsection{LSPIV data processing and post}

Dantec's FlowManager software was used to perform the necessary calibration, correlation functions, filtering, and statistical calculations on the images to generate the resolved velocity fields. Raw pixel displacement fields were computed using a standard cross-correlation algorithm. Interrogation windows were $32 \times 32$ pixels and were overlapped by $50 \%$ to increase the nominal resolution of the resultant pixel displacement grid to $16 \times 16$ pixels. The search windows were $64 \times 64$ pixels in size to ensure that all the particles in the interrogation window remained in the search window (Keane and Adrian, 1992). The first step of the post-processing involved identification and correction of spurious 
displacement vectors using a local-median scheme. Several thousand images were captured and processed into instantaneous velocity vector fields. The particle image diameter was estimated to be 2.2 pixels, which is close to the value of 2.0 pixels recommended by Raffel et al. (1997) to minimize peak locking. During the image acquisition, the LSPIV parameters were optimized to satisfy the condition that the maximum particle displacement be less than one-quarter of the interrogation area (IA). Using FlowManager's cross-correlation scheme, the necessary timing sequence to capture the flow field was established. The timing sequence was defined as the time interval between the two sequentially recorded images, commonly known as the image pair, required to resolve a single velocity field. If the correlation was poor, then the time interval was adjusted appropriately, a new sample image was recorded, and the timing sequence was again evaluated. This iterative procedure was repeated until a satisfactory timing sequence was achieved. The condition for satisfactory timing was a particle displacement not exceeding $25 \%$ of the interrogation area. This ensured that the image capture rate was sufficient to record the particle displacement within any interrogation area. With a satisfactory timing sequence established, a set of 500 image pairs was then recorded. Using FlowManager's cross correlation scheme, the recorded image pairs were correlated to produce velocity vector fields. To remove outliers from the velocity field, a moving-average filter, with a $3 \times 3$ neighborhood size, was used in the FlowManager. A temporally averaged velocity field of the 1000 image sample size was then obtained using FlowManager's statistics scheme.

\subsubsection{Filtering techniques}

Filtering the correlation can improve the signal to noise ratio, since it effectively reduces camera and light noise. The accuracy of the calibration depends on the quality of the calibration images. In the preliminary stage of the experiments, pictures were filtered using four different data filtrations techniques: (1) signal to noise ratio; (2) global; (3) local median; (4) interpolate outliers. A validation method in FlowManager based on a signal-to-noise ratio filter (SNR) is first used in the post-processing of PIV raw data to validate the vector field. SNR filtration discards all vectors when the ratio of the tallest peak in the correlation plane to the second tallest one is less than 1.3 (Kean and Adrian, 1992). A global filter is used to eliminate the vectors that are different from the rest of the vector field. If a vector is significantly larger or smaller than a majority of the vectors, then it is removed from the data. After global filtering, a local median filter is applied to the remaining vectors. A local median filter sorts velocities based on the squared difference between individual velocity vectors and the median of the mean of their surrounding neighbors. It takes individual vectors and compares them with their closest neighbors. The median of the vectors can easily be replaced with the mean value. Finally, an interpolation of the outliers is applied to local median-filtered vectors. Image-preprocessing can also affect the accuracy.

\subsubsection{Seeding particles}

The total error in PIV data is a function of out-of-plane particle motion, particle size, particle density, peak locking, the dynamic range of measured pixel displacements and the sub-pixel interpolation scheme employed (Cowen and Monismith, 1997). Seeding density impacts data quality and must be high enough so that an adequate number of seeding particles (two or more) are within the interrogation area between frames and low enough that pronounced particle agglomeration does not cause saturation of the interrogation area leading to measurement error (Raffel et al. 1997). Thus, data points were removed if agglomeration or inadequate tracer particles occurred in the image area. Raffel et al. (1997) showed that particle concentration influences the probability of detecting the valid displacement, as well as the measurement uncertainty. Tests with changing particle concentration showed that the number of particles in each interrogation area should be higher than five. The dispenser operation satisfies this criterion. Numerical analyses mentioned by Kean and Adrian (1990) lead to the conclusion that, if a flow can be densely seeded, both a high valid detection rate and a low measurement uncertainty can be achieved, especially when using small interrogation areas. 
2.2.3 LSPIV sensitivity to turbulence

As in traditional PIV, image quality is a key issue in LSPIV. The image scale and resolution are determined by the nature of the CCD sensor in the PIV camera and by the lens employed, and they vary with the scale of the flow under study. The instantaneous vector maps and the large number of instantaneous vector maps were used to calculate the mean velocity and turbulence quantities; the uncertainties in the mean velocity at $95 \%$ confidence level were estimated to be $2 \%$ of the local value, while those in the mean momentum flux and vorticity are 5\%. Averaging was done over 1000 PIV images.

\section{Experimental Setup and Procedure}

\subsection{Facility and operating conditions}

The experimental setup (Figure $3 \mathrm{a} \& \mathrm{~b}$ ) consists of a rectangular inlet channel $0.25 \mathrm{~m}$ wide and 1.0 $\mathrm{m}$ long, a rectangular shallow basin with inner dimensions of $6.0 \mathrm{~m} \times 4.0 \mathrm{~m}$ width, and a $0.25 \mathrm{~m}$ wide and $1.0 \mathrm{~m}$ long rectangular outlet channel. The water level in the basin is controlled by a $0.25 \mathrm{~m}$ wide and $0.30 \mathrm{~m}$ high flap gate at the end of the outlet. The basin is $0.30 \mathrm{~m}$ deep and has a flat bottom. The walls and bottom are hydraulically smooth. Adjacent to the basin, a mixing tank is used to prepare the water-sediment mixture. Along the basin side walls, a $4.0 \mathrm{~m}$ long, movable, aluminum frame is mounted, which carries the measurement instruments. Several variables were measured during every test: 2D surface velocities, thickness of deposited sediments, concentration of sediment inflow and outflow, water level in the basin, and discharge. Floating white polypropylene tracer particles with a diameter of $3.4 \mathrm{~mm}$, contrasted with the dark bottom, are used to visualize the surface velocity field. Instantaneous velocity fields are obtained by a digital camera connected to a computer and the PIV algorithm of FlowManager@.

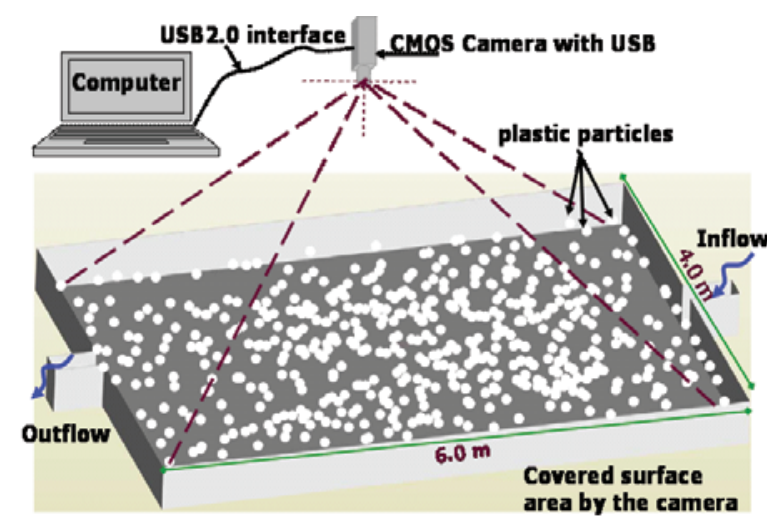

(a)

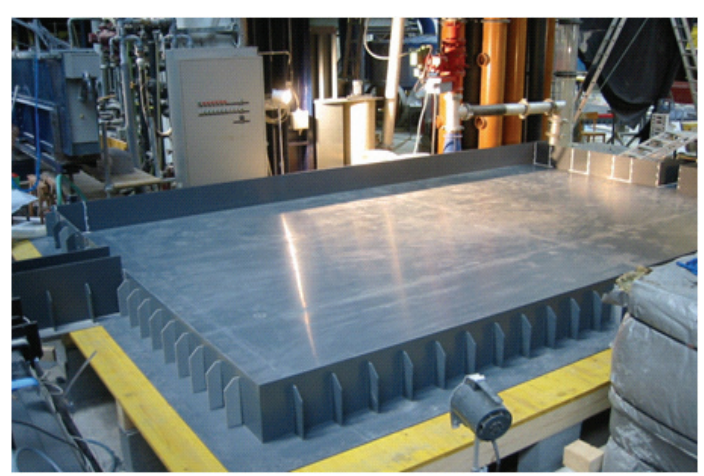

(b)

Fig. 3. (a) Scheme of LSPIV installations and data acquisition (b) Picture of the basin

Table 1. Hydraulic conditions of some of experiments.

\begin{tabular}{|l|l|l|l|l|}
\hline \multicolumn{1}{|c|}{ Test No } & \multicolumn{1}{c|}{$h[\mathrm{~m}]$} & $Q[\mathrm{l} / \mathrm{s}]$ & \multicolumn{1}{|c|}{$\operatorname{Fr}[-]$} & \multicolumn{1}{c|}{$\operatorname{Re}[-]$} \\
\hline $1,2,3,4,5$ & 0.2 & 7.0 & 0.1 & 28000 \\
\hline 5 & 0.15 & 7.0 & 0.157 & 27900 \\
\hline 5 & 0.1 & 7.0 & 0.283 & 28000 \\
\hline 5 & 0.075 & 7.0 & 0.435 & 27975 \\
\hline 6 & 0.2 & 3.5 & 0.05 & 14000 \\
\hline
\end{tabular}

\subsection{Configurations and Procedure for Tests}

To obtain a correctly modeled shallow turbulent free surface flow under laboratory conditions, a sufficiently high Reynolds number is applied, which generally results in a prohibitively large 
reservoir. Table 1 shows the hydraulic conditions of experiments with rectangular geometry. A reference basin geometry with a constant width of $\mathrm{B}=4.0 \mathrm{~m}$ and length of $\mathrm{L}=6.0 \mathrm{~m}$ is compared to the different test configurations, as summarized in Table 2. For the present paper, the following parameters remained constant for all configurations: discharge $(Q=7.0 \mathrm{l} / \mathrm{s})$, water depth $(h=0.2 \mathrm{~m})$, inlet and outlet channel dimensions (length $l=1.0 \mathrm{~m}$ and width $b=0.25 \mathrm{~m}$, horizontal bed slope, Froude number $(F r=0.1)$, and Reynolds number $(R e=28000)$. The different test configurations allow detailed study of the effect of geometry on the degree of asymmetry of the separated flow in the basin. After filling the basin and reaching a stable flow state with the clear water, LSPIV recordings were performed for 3 minutes. The geometrical parameters are defined, and all tests are summarized in Table 2. In order to represent all geometric parameters with flow results, a geometry shape factor $f$ was defined as $f=\left(A / P^{2}\right)^{*} E R$. In the present study, several reservoir geometries with different shapes have been investigated. Thus, there is a need for a dimensionless coefficient representative of different geometries that can be correlated with flow characteristics. The following definitions are used (see Table 1): length and the width of the basin: $\mathrm{L}$ and $\mathrm{B}$; lateral expansion ratio: $E R=\mathrm{B} / b$; aspect ratio as $A R=\mathrm{L} / \mathrm{B}$; the total surface area $A=\mathrm{L}^{*} \mathrm{~B}$ for rectangular basin; and $P$, the wetted perimeter of the length of the side walls $P=\sum_{i=1}^{n} a_{i}$, where $a_{i}$ is the length of the $\mathrm{i}$-th $(1 \mathrm{st}, 2 \mathrm{nd}, 3 \mathrm{rd} \ldots$ $\mathrm{n}$-th) side of an n-sided geometry.

Table 2. Configurations of different test series and their geometrical characteristics: $L$ and $B$ are length and width, $A$ the total surface area of the basin, $P$ is the wetted perimeter of the length of the side walls, and $E R$ and $A R$ are the expansion and aspect ratios. Shape factor is defined as $f=\left(A / P^{2}\right)^{*} E R$.

\begin{tabular}{|c|c|c|c|c|c|c|}
\hline Test No & $\mathrm{B}[\mathrm{m}]$ & $\mathrm{L}[\mathrm{m}]$ & $E R=\mathrm{B} / b[-]$ & $A R=\mathrm{L} / \mathrm{B}[-]$ & $f=\left(A / P^{2}\right) * E R[-]$ & Form \\
\hline $\begin{array}{l}1,2,3,4, \\
5,6\end{array}$ & 4.0 & 6.0 & 16 & 1.5 & 0.96 & \\
\hline 7 & 3.0 & 6.0 & 12 & 2.0 & 0.67 & \\
\hline 8 & 2.0 & 6.0 & 8 & 3.0 & 0.375 & \\
\hline 9 & 1.0 & 6.0 & 4 & 6.0 & 0.122 & \\
\hline 11 & 4.0 & 5.0 & 16 & 1.25 & 0.99 & \\
\hline 12 & 4.0 & 4.0 & 16 & 1.0 & 1.0 & \\
\hline 13 & 4.0 & 3.0 & 16 & 0.75 & 0.97 & \\
\hline 14 & 4 & 6 & 8 & 1.5 & 0.95 & \\
\hline 15 & 4 & 6 & 16 & 1.5 & 0.75 & \\
\hline 16 & 4 & 6 & 12 & 1.5 & 1.71 & \\
\hline
\end{tabular}




\section{Results and Discussion}

\subsection{Observed flow patterns and features}

Figure 4 shows an overview of the streamlines and behavior of large-scale coherent structures for two shapes with shape factors $f=\left(A / P^{2}\right) * E R$ of 0.96 and 0.99 (see also Table 2). For $f \leq 0.96$, a plane jet issues from the narrow leading channel and enters straight into the first half-meter of the much wider basin. After jet issuance, the main flow tends to curve towards the right hand side over the next two meters, until it stagnates against the right wall as shown in Figure 4(a). The main flow separates from the right wall, inducing a zone of flow recirculation (1). After the jet touches the wall, a separation occurs and generates a large main stable eddy in the center of the basin rotating counterclockwise with size $\mathrm{L}_{\mathrm{r} 3}$ and $\mathrm{B}_{\mathrm{r} 3}$. When monitoring the flow structure, two small 'triangular' gyres are formed by rotating clockwise in the upstream corners of the basin with dimensions $\mathrm{L}_{\mathrm{r} 1}$ and $\mathrm{B}_{\mathrm{r} 1}$ along the right corner and with dimensions $\mathrm{L}_{\mathrm{r} 2}$ and $\mathrm{B}_{\mathrm{r} 2}$ along the left corner. The deflected jet works as a vortex shedding region between the main eddy in the center and the triangular one in the upstream right corner. Moreover, two mixing layers M1 and M2 can be observed between the main flow and both eddies (Figure 4(a)).

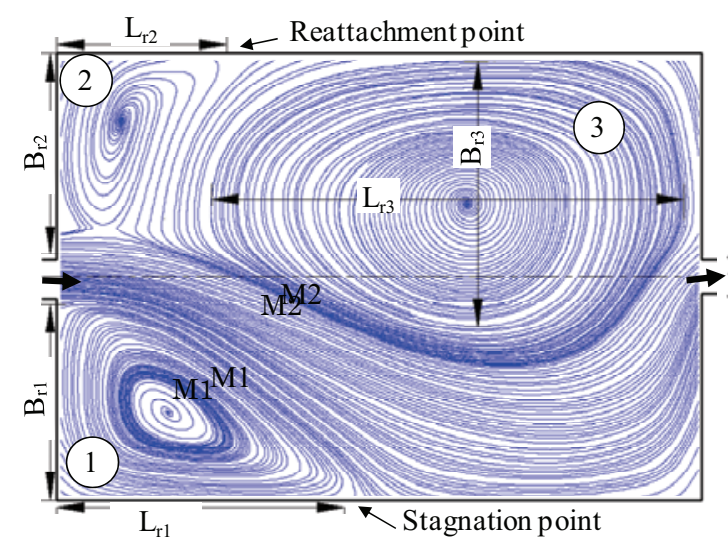

(a) $f=0.96$ (test 1, 2, 3 \& 4)

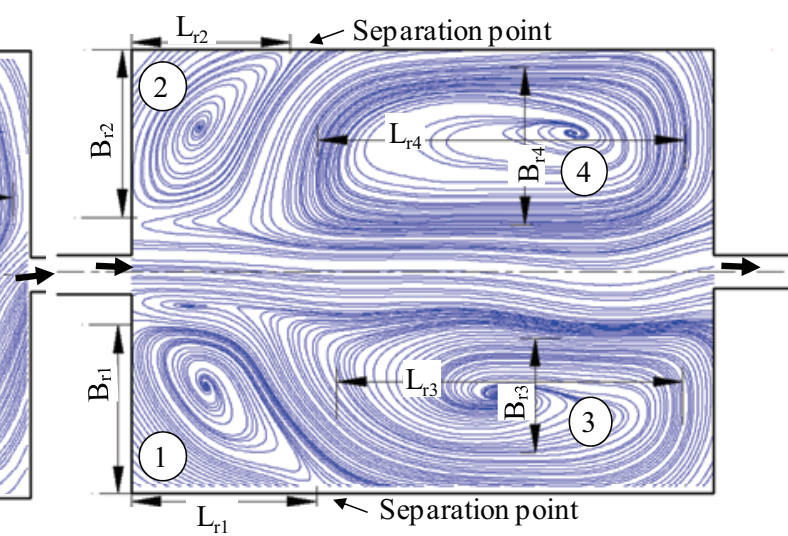

(b) $f=0.99$ (test 11)

Fig. 4 Time-averaged streamlines obtained by LSPIV measurements for clear water flow for two different basin geometries with shapes $f=\left(A / P^{2}\right) * E R$ of 0.96 and 0.99 . The geometrical parameters of the recirculation cells in the basin are $L_{r i}, B_{r i}$ for gyres $(1,2,3 \& 4)$.

The jet seems to be attracted to one side of the basin (in the tests, always to the right side). After the flow has reached the left wall by the counterclockwise large circulation, a separation point forms (Figure 4(a)). The streamline of the large gyre is shed from the separation point and connects with the core gyre. A second vortex shedding zone in the reverse direction is generated between the main gyre and the small triangular clockwise eddy in the upstream left corner. The reverse flow jet, which is generated by the inertia of the main gyre, pushes the incoming jet aside and forms a shedding point between five flow patterns: main flow jet, reverse flow jet, large main gyre, and right and left corner gyres. The jet preference for the right side is weak, since a stable mirror image of the flow pattern can easily be established by slightly disturbing the initial conditions. The stable asymmetric pattern, with a larger and smaller recirculation zone at the right and left corners, respectively, can be explained by a Coanda effect, by which any perturbation of the flow field pushing the main flow to one side of the basin gives rise to larger velocities. Thus, the asymmetry will naturally tend to be accentuated by this Coanda effect. By increasing the shape factor to $f>0.96$, the flow becomes more stable and symmetric, which will be explained in detail later. Figure 4(b) shows the second flow behavior that developed with a shorter geometry length (Tests No 11, 12 \& 13) and a hexagonal geometry (Test No 16). In that case, the flow became more stable and symmetric with four large vortices (4 regions in Figure 4(b)). By further increasing $f$ to 0.97 , the number of symmetric gyres is reduced to two (coupled) circulation cells along the centerline. The upstream corner vortices disappear completely. In conclusion, in the tested axisymmetric rectangular configurations, the flow 
is symmetric if $f>0.96$ and asymmetric if $f \leq 0.96$.

Flow patterns, streamlines, and mean vorticities for all test series are compared in Figures 5 and 6. For all tested geometries, two typical behaviors were observed. The first is a stable asymmetric flow pattern with one long and one short separation zone for reduced basin widths B (Test 11). The second is a symmetric flow pattern along the centerline with large scale vortices on both the right and left sides for reduced basin lengths and hexagonal geometries.

\subsection{Asymmetric flow for geometry shape factor $f \leq 0.96$}

Figure 5 (a) - (f) shows the flow behavior for various geometries with $f=0.96,0.67,0.375,0.122,0.75$ and 0.95 , respectively. On the left side of the figure, the flow patterns are shown by the velocity vectors and magnitude.

$[\mathrm{m} / \mathrm{s}]$
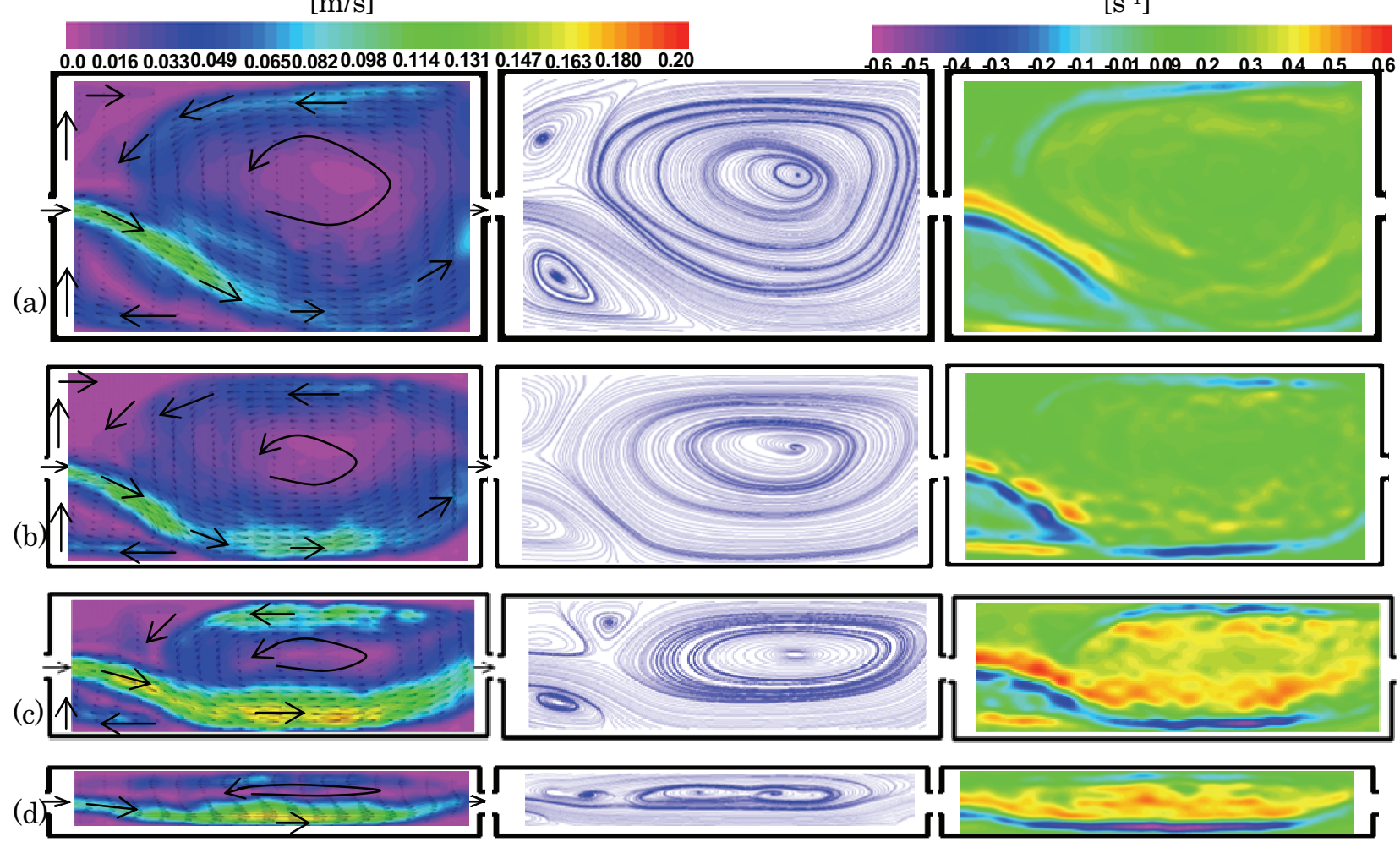

(e)

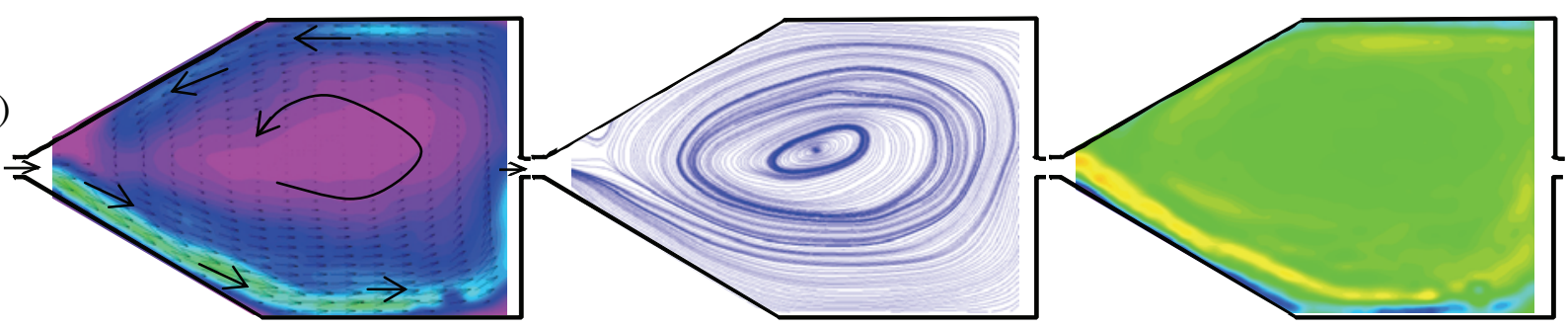

(f)

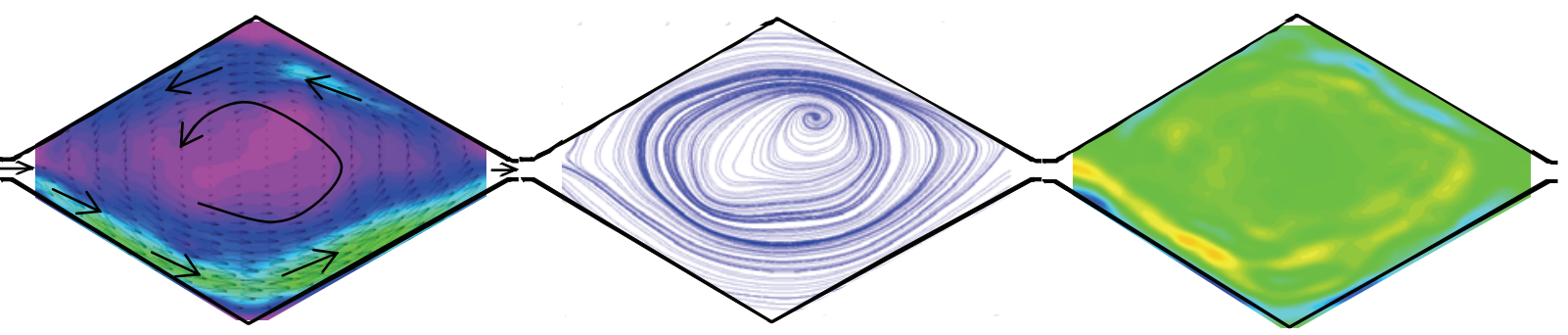

Fig. 5 Average flow pattern with velocity vectors (left), streamlines (middle) and mean vorticity (right) for six different geometries with shape factors $f=0.96(\mathrm{a}), 0.67$ (b), $0.375(\mathrm{c}), 0.122(\mathrm{~d}), 0.75(\mathrm{e})$, and 0.95 (f). 
Of particular interest is the effect of the geometry on the flow field and the separation zone on both sides. In Figure 5(a), the flow has an asymmetric behavior, leading to a larger gyre size in the right upstream corner side than in the left side. The main gyre size is in accordance with the two corner gyres. The two corner gyres are in accordance with each other and alternately change in size. Moreover, they control the size and location of the main gyre. By removing the upstream corners, as shown in Figure 5(e), the two corner gyres disappear, and only the core gyre is formed. No changes occur by removing the downstream corners in the diamond geometry shape. The observed flow pattern in Figure 5(f) did not differ much from what was previously explained for Figure 5(e), except for the reduction in size of the center gyre and downstream-shifted separation and reattachment points. The size and locations of the three vortices are clearly visible by streamlines shown in the middle of Figures 4 (a), (b), (c), (d), (f) and (g). On the right side of the same figures, the mean vorticities of the flows are shown.

$$
[\mathrm{m} / \mathrm{s}]
$$

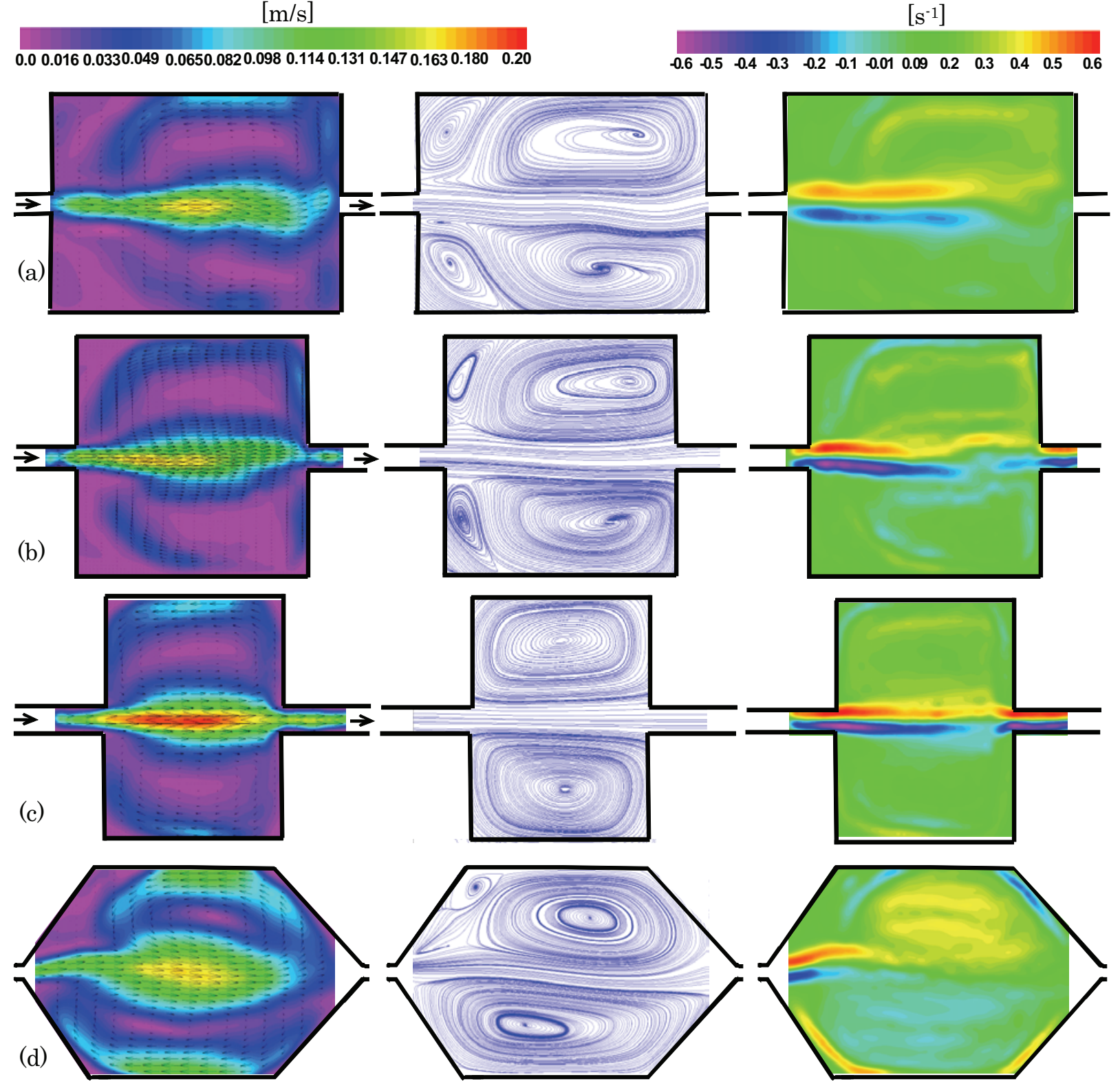

Fig. 6 Average flow pattern with velocity vectors (left), streamlines (middle) and mean vorticity (right) for four different geometries with shape factors $f=0.97(\mathrm{a}), 0.98$ (b), 0.99 (c), and 1.71 (d). 
The vorticity is defined as the curl of the velocity vector. In accordance with the flow patterns and streamlines, the high vorticity is concentrated at the curved jet entering from the inlet channel and the right corner. Figure 5(c) exhibits a relatively large area with strong vorticity. The vorticity reflects a large portion of flow stabilization due to width reduction. By reducing the basin width, the flow becomes more homogenous and the vorticity increases. Moreover, no stagnant water is observed in the center of the gyres shown in Figure 5(c) \& (d).

\subsection{Symmetric flow for geometry shape factor $f>0.96$}

Asymmetry disappears when the geometry shape factor is higher than 0.96, as illustrated by Figure 6 (a), (b), (c) and (d) for LSPIV results for shape factors $f=0.97,0.98,0.99$ and 1.71, respectively. By reducing the basin length, and consequently increasing $f$ ratios, the flow is stabilized with a stable symmetrical pattern. Four vortices exist in the basin for an $f$ ratio larger than 0.97 , as shown in the middle illustration in Figure 6(a). The four gyres interact with the jet, which has some tendency to meander. By reducing the basin length for $f=1$, the number of gyres remains constant, and the flow pattern becomes rather symmetric with respect to the centerline (middle, Figure 6(b)). Completely symmetric streamlines with two vortices are exhibited in Figure 6(c) middle, for $f$ ratio of 0.97 and a basin length less than its width.

Similar flow patterns and streamlines with two vortices occur by removing the four corners of the reference basin with an $f$ ratio of 1.71 (Figure 6(d)). The predominant change in the flow pattern is an evolution from a four-vortex flow to a distinct two-vortex flow. The corresponding vorticities for these streamlines are shown in Figures 5(a), (b), (c) and (d), on the right. Comparison of these figures shows that the vorticity values are increasing with decreasing basin lengths. Comparing all geometries with $f>0.96$ reveals that similar symmetric flow patterns occur with small differences regarding the dimensions and strengths of the circulation cells.

\section{Conclusion}

Shallow flows are predominantly two-dimensional flow structures, which means that the overall behavior of the flow can be analyzed using surface velocities. To obtain a correctly modeled shallow turbulent free surface flow in a laboratory, a sufficiently high Reynolds number and a sufficiently low Froude number are required, which generally necessitate large facilities where the horizontal dimensions are in the order of meters. The experiments described in this paper were conducted with the goal of demonstrating the capabilities of LSPIV in the measurements of $2 \mathrm{D}$ surface flow for shallow basins with different configurations. Furthermore, they should allow understanding of the effects of the geometry of shallow basins on flow behavior.

The flow structures in a shallow basin can be measured successfully by using LSPIV. It could be demonstrated that LSPIV has potential for measuring low velocities. The influence of the geometric parameters expressed by the shape factor $f$ was examined for symmetric inflow and outflow conditions. Although the geometry is symmetric, the flow pattern becomes asymmetric under certain conditions. For $f \leq 0.96$, the flow changes from a symmetric structure to an asymmetric structure. The basin length has a strong influence on changing the flow field from an asymmetric flow to a stable symmetric flow. The basin width did not influence the asymmetric separation of the issuing jet. However, the size of the main and secondary eddies were in accordance with the width. It can be concluded that the basin geometry influences the behavior of the large turbulence structures, and the flow is quite sensitive to the geometry shape factor.

LSPIV measurements at the water surface, using an appropriate camera and homogeneously particle spreading of sufficient density, provide a suitable method to analyze the behavior of such flows with different geometries. Using the water surface as a measurement plane, normal lights have to be used. Any other application where the surface velocity represents the behavior of the overall flow, because the flow is quasi two-dimensional, such as shallow wake flows or confluence problems in rivers, can be analyzed using the presented technique. 


\section{References}

Adamsson, A., Stovin, V., and Bergdahl, L., Bed shear stress boundary condition for storage tank sedimentation, J. Environ. Eng.-ASCE, 129 (2003), No 7, 651-658.

Adrian, R. J., Particle-imaging techniques for experimental fluid mechanics, Ann. Rev. Fluid Mech., 23 (1991), 261-268

Chen, D. and Jirka, G., Absolute and convective instabilities of plane turbulent wakes in a shallow water layer. J Fluid Mech 338 (1997), 157-172

Dewals, B. J., Kantoush, S. A., Erpicum, S., Pirotton, M., and Schleiss, A. J., Analysis of flow instabilities in shallow rectangular basins, Environmental Fluid Mechanics, 8 (2008), No 31, 31-54.

Dersch, H., Dersch Helmu's original home page. http://webuser.fh-furtwangen.de/ dersch/, (2003).

Emttea, R., Fujita, I., Muste, M. and Krufer, A., Particle Image Velocimetry for whole field measurement of ice velocities. Cold Regions Science and Technology, 26 (1997), 97-112.

Fujita, I. and Komura, S., Application of video image analysis for measurements of river-surface flows, Proc. Hydraul. Eng., JSCE 38 (1994), 733-738.

Fujita, I., Muste, M. and Kruger, A., Large scale Particle Image Velocimetry for flow analysis in hydraulic engineering applications, Journal of hydraulic Research, 36 (1998), No 3, 397-414.

Kantoush, S.A., Bollaert, E.F.R., Boillat, J.-L. and Schleiss, A.J., Suspended load transport in shallow basins, XXXI IAHR conference proceeding, September (2005), Seoul, South Korea

Kantoush, S.A., Bollaert, E.F.R., Boillat, J.-L., Schleiss, A.J., and Uijttewaal, W.S.J., Sedimentation Processes in Shallow basins with different geometries, IAHR Proc. of the International Conference on Fluvial Hydraulics, Lisboa, Portugal, (2006), 1623-1631.

Kantoush, S. A., Symmetric or asymmetric flow patterns in shallow rectangular basins with sediment transport, 32nd Congress of IAHR, John F. Kennedy student competition, 1-6 July, (2007), Venice, Italy.

Kantoush, S. A., De Cesare, G., Boillat, J.-L., and Schleiss, A. J. Flow field investigation in a rectangular shallow reservoir using UVP, LSPIV and numerical modeling. J. Flow Measurement and Instrumentation, (2008), Vol. 19(3-4), PP. 139-144

Kantoush, S. A., Bollaert, E., and Schleiss, A. J., Experimental and numerical modelling of sedimentation in a rectangular shallow basin, International Journal of Sediment Research, Vol. 23, No. 3, (2008), pp. 1-26.

Kantoush, S. A., and Schleiss, A. J., Channel Formation in Large Shallow Reservoirs with Different Geometries during Flushing, Journal of Environmental Technology, 30 (2009), No. 9, 1020-1032

Muste, M., Xiong, Z., Schöne,J. and Li, Z., Flow diagnostic in hydraulic modeling using image velocimetry, J. Hydraul. Eng. 130 (2004), No 3, 175-185.

Raffel, M., Willert, C. and Kompenhaus, J., Particle Image Velocimetry, Springer-Verlag (1997)

Shapira, M., Degani, D. and Weihs, D., Stability and existence of multiple solutions for viscous flow in suddenly enlarged channels, Comput. Fluids, 18 (1990), No 3, 239-258.

Uijttewaal, W.S.J., Lehmann, D. and Mazijk, A. van, Exchange processes between a river and its groyne fields: Model experiments, J. Hydr. Engrg.127 (2001), 928-936.

Willert C., Stereoscopic digital particle image velocimetry for application in wind tunnel flows, Meas Sci Technol 12(1997), 1465- 1479

Weitbrecht, V., Kühn, G. and Jirka, G.H., Large scale PIV-measurements at the surface of shallow water flows, Flow Meas. and Instr. 13 (2002), 237-245.

Yuce, M. I. and Chen, D., An experimental investigation of pollutant mixing and trapping in shallow costal re-circulating flows. In Proc. the Int. Symp. On shallow flows, (2003) Part I:165-172, Delft, The Netherlands.

\section{Author Profile}

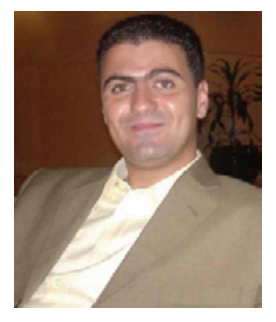

Sameh Ahmed Kantoush: completed his undergraduate studies at Alexandria University in the Civi Engineering Dept., Egypt, in 1997. After graduation, he worked in academic (Alexandria University and Arab Academy) and practical fields (Consulting Engineering Office and construction companies) for three ears. From 2001 to 2003, he attended Saga University, Faculty of Science and Engineering, Japar throe jo Japan, Laboratoire de Constructions Hydrauliques (LCH) at the Swiss Federal Institute, Switzerland, from 2003 to 2008, as a PhD student and research associate, he obtained Doctorate on the topic of reservoir sedimentation. He has been awarded a Swiss National Fund for Scientific Research (SNSF) awarded for a post doctoral research at Water Resources Research Centre, Disaster Prevention Research Institute, Kyoto University, Japan, in 2009.

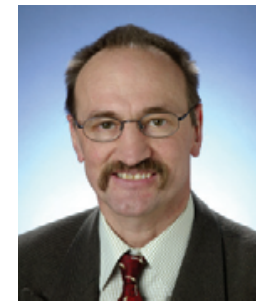

Prof. Dr. Anton J. Schleiss graduated from the Swiss Federal Institute of Technology (ETH) in Zurich, Switzerland, in 1978 with a degree in Civil Engineering. After joining the Laboratory of Hydraulic, Hydrology and Glaciology at ETH as a research associate and senior assistant, he obtained a Doctorate of Technical Sciences on the subject of pressure tunnel design in 1986. After that, he worked for 11 years for Electrowatt Engineering Ltd. and was involved in the design of many hydropower and water resources projects. Until 1996, he was Head of the Hydraulic Structures Section in the Hydropower Department at Electrowatt. In 1997, he was nominated for full professor and became Director of the Laboratory of Hydraulic Constructions (LCH) in the Civil Engineering Department of the Swiss Federal Institute of Technology Lausanne (EPFL). He is also involved as an international expert in several dam and hydropower plant projects all over the world, as well as flood protection projects mainly in Switzerland. In April 2006, he became Director of the Civil Engineering Department of EPFL. He is President of the Swiss Committee of Dams and also of the Swiss Committee of Flood Protection. 